\title{
A comparative analysis of KMT2D missense variants in Kabuki syndrome, cancers and the general population
}

DOI:

10.1038/s10038-018-0536-6

\section{Document Version}

Accepted author manuscript

Link to publication record in Manchester Research Explorer

\section{Citation for published version (APA):}

Faundes, V., Malone, G., Newman, W. G., \& Banka, S. (2019). A comparative analysis of KMT2D missense variants in Kabuki syndrome, cancers and the general population. Journal of Human Genetics, 64(2), 161-170. https://doi.org/10.1038/s10038-018-0536-6

\section{Published in:}

Journal of Human Genetics

\section{Citing this paper}

Please note that where the full-text provided on Manchester Research Explorer is the Author Accepted Manuscript or Proof version this may differ from the final Published version. If citing, it is advised that you check and use the publisher's definitive version.

\section{General rights}

Copyright and moral rights for the publications made accessible in the Research Explorer are retained by the authors and/or other copyright owners and it is a condition of accessing publications that users recognise and abide by the legal requirements associated with these rights.

\section{Takedown policy}

If you believe that this document breaches copyright please refer to the University of Manchester's Takedown Procedures [http://man.ac.uk/04Y6Bo] or contact uml.scholarlycommunications@manchester.ac.uk providing relevant details, so we can investigate your claim.

\section{OPEN ACCESS}




\section{A comparative analysis of $K M T 2 D$ missense variants in Kabuki}

\section{2 syndrome, cancers and the general population}

3 Víctor Faundes $^{1,2}$, Geraldine Malone ${ }^{3}$, William G. Newman ${ }^{1,3}$, Siddharth Banka ${ }^{1,3}$.

5 1. Division of Evolution \& Genomic Sciences, School of Biological Sciences, Faculty of

6 Biology, Medicine and Health, University of Manchester, Manchester M13 9WL, UK.

7 2. Laboratorio de Genética y Enfermedades Metabólicas, Instituto de Nutrición y Tecnología

8 de los Alimentos (INTA), Universidad de Chile, Santiago 7830490, Chile.

9 3. Manchester Centre for Genomic Medicine, St Mary's Hospital, Manchester University

10 NHS Foundation Trust, Manchester M13 9WL, UK.

12 Running title: Comparative analysis of KMT2D missense variants.

13 The authors declare no conflicts of interest.

\section{Correspondence}

16 Dr Siddharth Banka

17 Manchester Centre for Genomic Medicine

18 St Mary's Hospital

19 Manchester M13 9WL

20 United Kingdom

21 Tel: +44 (0) 16170 10980; Fax: +44 (0) 1612766145

22 siddharth.banka@manchester.ac.uk. 


\section{Abstract}

26 Determining the clinical significance of germline and somatic $K M T 2 D$ missense variants

27 (MVs) in Kabuki syndrome (KS) and cancers can be challenging. We analysed 1920 distinct

28 KMT2D MVs that included 1535 germline MVs in controls (Control-MVs), 584 somatic

29 MVs in cancers (Cancer-MVs) and $201 \mathrm{MV}$ in individuals with KS (KS-MVs). The

30 proportion of MVs likely to affect splicing was significantly higher for Cancer-MVs and KS-

31 MVs than in Control-MVs $(\mathrm{p}=0.000018)$. Our analysis identified significant clustering of

32 Cancer-MVs and KS-MVs in the PHD\#3 and \#4, RING\#4 and SET domains. Areas of

33 enrichment restricted to just Cancer-MVs (FYR-C and between amino acids 3 043-3 248) or

34 KS-MVs (Coiled-coil\#5, FYR-N and between amino acids 4 995-5 090) were also found.

35 Cancer-MVs and KS-MVs tended to affect more conserved residues (lower BLOSUM scores, $36 \mathrm{p}<0.001$ and $\mathrm{p}=0.007)$. KS-MVs are more likely to increase the energy for protein folding

37 (higher ELASPIC $\Delta \Delta \mathrm{G}$ scores, $\mathrm{p}=0.03$ ). Cancer-MVs are more likely to disrupt protein

38 interactions (higher StructMAn scores, $p=0.019$ ). We reclassify several presumed pathogenic

39 MVs as benign or as variants of uncertain significance. We raise the possibility of as yet

40 unrecognised 'non-KS' phenotype(s) associated with some germline pathogenic KMT2D

41 MVs. Overall, this work provides insights into the disease mechanism of $K M T 2 D$ variants

42 and can be extended to other genes, mutations in which also cause developmental syndromes

43 and cancer.

45 Keywords: KMT2D; Kabuki syndrome; missense variant; protein domain 


\section{$46 \quad 1$ Introduction}

47 Histone lysine methylation defects are an important cause for developmental disorders and

48 cancers $(1,2) . K M T 2 D$ (formerly known as $M L L 2$ and $A L R$ ) encodes lysine (K)-specific

49 methyltransferase 2D, which catalyses the mono-, di- and trimethylation of the lysine 4 on

50 histone $3(\mathrm{H} 3 \mathrm{~K} 4)$, promoting the expression of its target genes (3). Germline deleterious

51 heterozygous $K M T 2 D$ variants cause Kabuki syndrome type 1 (KS, MIM\# 147920), a rare

52 congenital disorder characterized by intellectual disability, growth retardation, distinctive

53 facial features and structural anomalies (4-7). Somatic deleterious KMT2D variants have been

54 described in a spectrum of cancers including leukaemias, gastrointestinal and central nervous

55 system tumours $(8,9)$.

57 Correct interpretation of $K M T 2 D$ variants is crucial for diagnosis in $\mathrm{KS}$ and disease

58 progression in cancers $(10,11)$. About $80 \%$ of deleterious germline $K M T 2 D$ variants are

59 predicted to result in a truncated protein (5) (Figure S1). Germline pathogenic missense

$60 K M T 2 D$ variants are also frequently encountered in $\mathrm{KS}(4,5,12-31)$. In contrast, only $35 \%$ of

61 somatic KMT2D variants in cancers are predicted to be protein truncating (Figure S1).

62 Approximately $50 \%$ of the somatic variants found in cancers are missense, and the remaining

63 are in-frame insertions/deletions and synonymous variants (32) (Figure S1).

65 Although limited functional analysis of $K M T 2 D$ variants is now possible, determining the

66 consequences of $K M T 2 D$ missense variants (MVs) in diagnostic setting remains challenging

67 because parental segregation is not always possible, and especially due to incomplete

68 understanding of KMT2D protein structure and its interactions (33-36). Notably, the three-

69 dimensional structure of only the SET domain of the protein is available (PDB entries 4z4p

70 and 4erq) (37). A systematic study of KMT2D MVs can, therefore, have significant clinical 
71 benefits and help to distinguish pathogenic from benign germline variants and driver somatic

72 variants from passenger ones. Additionally, this may provide insights into the structure and

73 function of this important protein. Furthermore, the consequences of disease-causing

74 germline and somatic variants can be different. For example, some activating somatic $B R A F$

75 variants cause malignant melanoma (38), while other activating germline $B R A F$ variants

76 cause cardiofaciocutaneous syndrome (MIM \#115150) (39). Somatic loss-of-function

77 SMARCA4 variants cause hypercalcemic type small cell carcinoma of the ovary (40) and

78 postulated activating germline SMARCA4 variants are associated with Coffin-Siris syndrome

79 (MIM \#614609) (41). However, germline and somatic KMT2D MVs have not previously

80 been systematically compared. Likewise, loss-of-function, dominant negative or activating

81 germline MVs in the same gene can cause different phenotypes or diseases (42-45).

82 Although, all KS-causing $K M T 2 D$ variants are presumed to be loss-of-function, the

83 possibility of other phenotypes resulting from a different spectrum of germline $K M T 2 D$

84 variants has not been examined. Similarly, loss-of-function, dominant negative or activating

85 somatic MVs can have different consequences (46). However, this aspect has not been

86 explored for KMT2D previously. For all these reasons, we performed a comprehensive

87 systematic study of KMT2D MVs.

88

$89 \quad 2$ Methods

90 The study design is summarised in Figure 1. The databases and tools used in this study are

91 summarised in Tables S1 and S2.

\section{$92 \quad 2.1$ Compilation and interpretation of KMT2D MVs}


$93 K M T 2 D$ MVs reported in control population (Control-MVs) were compiled from the Exome

94 Aggregation Consortium (47) (ExAC, Version 0.3.1) database, the 1000 Genomes (1K-G)

95 Project (48), Database of Single Nucleotide Polymorphisms (dbSNP) (49) and the NHLBI-

96 GO Exome Sequencing Project (ESP) (50). The ExAC data was accessed via

97 http://exac.broadinstitute.org/ and the other data were obtained from the Ensembl version 80-

98 GRCh37. For ExAC, only high-quality and non-flagged sites were included. For analyses, we 99 assumed that Control-MVs did not result in any phenotype.

$101 K M T 2 D$ MVs annotated as being identified only in somatic tissue (Cancer-MVs) were 102 compiled from the Catalogue of Somatic Mutations in Cancer (COSMIC) (32) database, 103 version 77.

$105 K M T 2 D$ MVs reported in KS (KS-MVs) were obtained from literature (and cross-checked 106 with Human Gene Mutation Database Professional ${ }^{\circledR}$ [HGMD]) (51), ClinVar (52) and our 107 in-house database for Kabuki syndrome test results. Of note, the Manchester Centre for

108 Genomic Medicine has offered diagnostic KMT2D genotyping by sequencing since 2012. 109

110 All the Control-MVs, Cancer-MVs and KS-MVs were assessed by the Ensembl Variant

111 Effect Predictor (VEP) (53) to obtain their minor allele frequencies and to identify the

112 variants that were likely to disrupt splicing. EX-SKIP tool (54) was used to identify

113 substitutions that may result in exon skipping in mature transcripts. All MVs predicted not to

114 disrupt splicing were mapped with their frequencies on KMT2D protein domains, regions and

115 motifs (according to UniProt accession number O14686) using the Mutation Mapper tool

116 from the cBio Cancer Genomics Portal $(55,56)$. For purpose of our analysis, we divided the 
117 regions of the protein sequence that are not part of a specific domain or motif into 19 'no

118 domain' regions (Figure S2).

119

120 Next, for all MVs that were predicted not to significantly affect splicing, we generated the

121 Blocks Substitution Matrix Series 62 (BLOSUM62) (57) scores for evolutionary conservation

122 analyses, the Ensemble Learning Approach for Stability Prediction of Interface and Core

123 mutations (ELASPIC) algorithm $\Delta \Delta G$ values (58) for changes to the thermodynamic

124 properties resulting from substitutions, the Structural Mutation Annotation (StructMAn) score

125 (59) for calculating the impact of MVs on the interaction of KMT2D with other proteins and

126 ligands, obtaining the probability-of-disruption scores when possible. The PDB file for the

127 longest chain reported for KMT2D as part of a complex was downloaded from the Protein

128 Data Bank in Europe (37) (PDB entry 4erq) in order to support the analyses given by

129 ELASPIC and StructMAn.

130

$131 \quad 2.2$ Statistical Analysis

132 To study the association between the type of the phenotype and the location of MVs, the

133 likelihood ratio chi-square test was applied. The Z-test with the Bonferroni correction was

134 used to compare the proportion of MVs on each location according to the phenotype. The

135 Kruskal-Wallis test with multiple comparisons was applied to compare the BLOSUM62

136 scores, ELASPIC $\Delta \Delta G$ and StructMAn interaction scores amongst the phenotypes, which

137 were also described using the median and interquartile range. For all statistical analyses, the

138 IBM SPSS $®$ version 22 programme was used and a two-sided, exact p-value $<0.05$ was

139 considered as significant.

140 


\section{Results}

\section{$142 \quad 3.1$ Compilation of variants}

143 In total we identified 1920 distinct MVs, which included 1535 KMT2D Control-MVs, 584

144 KMT2D Cancer-MVs and $201 \mathrm{KS}-\mathrm{MVs}$ (Table S3). Of note, six MVs were reported in all

145 three groups, 85 were reported in both Cancer-MVs and Control-MVs groups, 83 were

146 reported in both KS-MVs and Control-MVs groups, and 23 were reported in both Cancer-

147 MVs and KS-MVs groups (Figure S3) (Table S3-1).

148

149 The MAFs for 1 211/1 535 (78.9\%) Control-MVs were $<1 / 10000$, and for 53/1 535 (3.5\%)

150 Control-MVs was >1/1 000 (Table S3). The Arg5048 was the most frequently altered amino

151 acid in the Cancer-MVs group (7/584, 1.2\%), followed by Arg3582 and Arg3727 (each

$1525 / 584,0.9 \%$ ) (Table S3). The Arg5179 was the most frequently altered amino acid in the KS-

153 MVs group (8/201, 4\%), followed by the Arg5048 and Arg5432 amino acids (each 7/201,

$154 \quad 3.5 \%)($ Table S3).

155

156 16/1 535 Control-MVs, 14/584 Cancer-MVs and 11/201 KS-MVs were predicted to

157 significantly affect splicing (two of these variants were present in both Control-MVs and KS-

158 MVs groups, and one in both Cancer-MVs and KS-MVs groups) (Table S3-2) (Figure 2). As

159 these variants are likely to result in loss of function by introduction of frameshift, they were

160 excluded from subsequent analyses that were performed on 1,519 Control-MVs, 570 Cancer-

161 MVs and $190 \mathrm{KS}-\mathrm{MV}$ s. The proportion of presumed MVs predicted to affect splicing is

162 significantly higher for KS-MVs and Cancer-MVs in comparison with Control-MVs

$163\left(\chi^{2}=21.88, \mathrm{df}=2, \mathrm{p}=0.000018\right)$. Of these 41 variants that are predicted to disrupt splicing, $6 / 16$

$164(37.5 \%)$ in controls, 8/14 (57.1\%) in cancer and 7/11 (63.6\%) in KS affect either the first or 
165 last bases of exons, demonstrating a further enrichment of canonical splice-donor and splice166 acceptor sites in cancer and KS (Table S3-2) (Figure 2). EX-SKIP tool analysis showed that

167 out of these six Control-MVs, two (c.50C $>$ T and c.5188G $>$ A) did not increase the probability

168 of exon-skipping when compared against wild-type (WT) and the remaining four

169 (c. $4131 \mathrm{G}>\mathrm{C}, \mathrm{c} .4419 \mathrm{G}>\mathrm{T}, \mathrm{c} .4693 \mathrm{G}>\mathrm{T}, \mathrm{c} .4694 \mathrm{C}>\mathrm{T}$ ) variants were predicted to result in in-

170 frame exon skipping.

171

$172 \quad 3.2$ Location of MVs

173 We identified several regions of constraint for Control-MVs (Figure 3; Tables 1 and 2).

174 Cancer-MVs clustered in PHD\#3, PHD\#4, RING\#4, FYR-C, and SET domains in

175 comparison with Control-MVs $(\mathrm{p}<0.05)$ (Tables 1 and 2). Cancer-MVs also clustered

176 specifically between amino acid numbers 3 043-3 248 (No Domain \#8 in Figure S2) when

177 compared with Control-MVs and KS-MVs $(\mathrm{p}<0.05)$ (Table 2). KS-MVs clustered in PHD\#3,

178 PHD\#4, Coiled-coil\#5, RING\#4, FYR-N and SET domains when compared with Control-

179 MVs $(\mathrm{p}<0.05)$ (Tables 1 and 2). KS-MVs also clustered specifically between amino acid

180 numbers 4 995-5 090 (No Domain \#16 in Figure S2) when compared with Control-MVs and

181 Cancer-MVs $(\mathrm{p}<0.05)$ (Table 2).

182

$183 \quad 3.3$ Consequences on protein properties

184 The median BLOSUM score for Control-MVs was $-1(-2 ; 1)$, for Cancer-MVs was $-1(-2 ; 0)$,

185 and for KS-MVs was -1 (-2;0) (Figure 4). Overall, the BLOSUM scores for Cancer-MVs and

186 KS-MVs were significantly lower when compared to Control-MVs $(\mathrm{p}<0.001$ and $\mathrm{p}=0.007$, 187 respectively) (Figure 4). 
189 The ELASPIC $\Delta \Delta G$ score for Control-MVs was $0.76(0.25 ; 1.07)$, for Cancer-MVs was 0.89

$190(0.4 ; 1.46)$, and for KS-MVs was $0.98(0.34 ; 2.17)$ (Figure 4). The ELASPIC $\Delta \Delta G$ scores for

191 KS-MVs were significantly higher when compared to Control-MVs $(\mathrm{p}=0.03)$ (Figure 4). No

192 other pairwise comparisons were significant (Figure 4).

193

194 The StructMAn score for Control-MVs was $0.17(0.14 ; 0.26)$, for Cancer-MVs was 0.32

$195(0.15 ; 0.42)$, and for KS-MVs was $0.21(0.14 ; 0.34)$ (Figure 4). The StructMAn scores for

196 Cancer-MVs were significantly higher when compared to Control-MVs $(p=0.019)$. No other

197 pairwise comparisons were significant (Figure 4).

\section{Discussion}

199 We present a comprehensive analysis of KMT2D MVs reported in control populations,

200 cancers and KS. Rare KMT2D MVs are frequent in the general population as nearly $80 \%$ of

201 Control-MVs have a MAF $<1 / 10000$ (Table S3). Hence, the rarity of a KMT2D variant is not

202 a reliable indicator of pathogenicity. This compilation highlights five arginine residues in

203 KMT2D that are recurrently substituted in cancer (Arg5048, Arg3582 and Arg3727) and KS

204 (Arg5048, Arg5179 and Arg5432) (Table S3). Interestingly, Arg5048 is amongst the most

205 frequently mutated residues in both cancer and in KS. Arg5048 and Arg5432 are located

206 outside any recognized domains of the protein (No domain \#16 and \#18, respectively in

207 Figure S2). The Arg5432Trp substitution has been shown to disrupt the interaction of

208 KMT2D with RBBP5 and ASH2L, and result in loss of its catalytic activity (60). Arg5179 is

209 located in the FYR-N domain, which is a region of around 50-100 amino acids enriched in

210 phenylalanine $(\mathrm{F})$ and tyrosine $(\mathrm{Y})$ found in chromatin-associated proteins (61). Arg3582 and

211 Arg3727 are located in the coiled-coils \#3 and \#4, respectively. Coiled-coils are a type of 
212 secondary structure composed of two or more alpha helices which pack together like a cable.

213 These structures help to position catalytic activities at fixed distance (62).

214

215 Intriguingly, we found that six KMT2D MVs have been described in controls, cancers and

216 KS; 85 in cancer and controls; and 83 in KS and controls (Tables S3-1). Several possibilities

217 could account for these MVs being observed in control and disease cohorts. Overlap between

218 controls and cancer MVs could be explained by incorrect curation of germline variants as

219 somatic-only in the COSMIC database or wrongly curated somatic variants as germline

220 benign variants in controls. Overlap between controls and KS-MVs could be explained by

221 incorrect interpretation of pathogenicity of these benign variants in KS. Alternatively, these

222 variants may be causing KS with reduced penetrance. However, incomplete penetrance has

223 never been reported in KS. Notably, in other disorders, somatic mosaicism of truly

224 pathogenic variants in healthy controls has been described (e.g. in Bohring-Opitz syndrome)

225 (63) and this could be another explanation for some overlap observed between KS-MVs and

226 Control-MVs. 65/83 of the overlapping KS-MVs and Control-MVs are located outside the

227 regions of enrichment in KS-MVs, therefore, they are more likely to be benign variants

228 (Table S3-1).

229

230 MVs predicted to alter splicing, those affecting canonical splice-donor and splice-acceptor

231 sites were significantly more frequent in cancer and KS, which is consistent with the loss-of-

232 function mechanism associated with these two disorders (Table S3-2) (Figure 2). These

233 variants in cancer and KS should be more appropriately reclassified as splicing variants.

234

235 Of note, the six Control-MVs affecting the first or last nucleotide of exons are all located at

236 the first half of the gene (exons 2, 13, 16, 17, 18, 21; Figure 2), which should allow the 
expression of an alternative protein coding transcript (ENST00000526209.1). The protein encoded by this alternative transcript includes the catalytic SET and Post-SET domains without the PHD-type and RING-type zinc fingers, the SPPPEPEA region, the HMG Box, coiled-coils, the LXXLL motifs and the FYR-N and $-\mathrm{C}$ domains (Figure S2). This observation points towards the potential redundancy of the N-terminus of KMT2D, which is consistent with previous observations and may indicate the compensatory capacity of the alternative transcript for normal development $(60,64,65)$. Interestingly, $11 / 16(68 \%)$

$244 K M T 2 D$ protein-truncating variants (PTVs) reported in ExAC are located in the first half of 245 the gene (from residue 1 to 2,768). This is in contrast with KMT2D PTVs in HGMD and 246 COSMIC, where $39 \%$ of KS-PTVs, and 53\% of Cancer-PTVs are in this region.

We demonstrate significant clustering of Cancer-MVs and KS-MVs in the PHD-type zinc fingers \#3 and \#4, RING-type zinc finger \#4 and SET domains, reflecting the importance of these domains in the function of KMT2D. The PHD (plant homeodomain) fingers are domains of 50-80 amino acids containing a zinc-binding motif that appears in many chromatin-associated proteins, which recognise methylated H3K4 (66). The RING-type zinc fingers are composed of 40-60 amino acids that bind two atoms of zinc, and may mediate protein-protein interactions (67). The SET ( $\underline{\operatorname{Su}}(\mathrm{var}) 3-9$, Enhancer-of-zeste, and Trithorax) domain is composed of 130-140 amino acids in which resides the methyltransferase activity and the substrate-binding sites $(60,68)$. This similarity of clustering seen in Cancer-MVs and KS-MVs is strongly suggestive that these variants result in loss-of-function.

We found significant clustering of Cancer-MVs in the FYR-C domain and between residue numbers 3 043-3 248 (No domain \#8 in Figure S1). The FYR-C domains have the features

261 similar to those of FYR-N domains (61). Notably, these regions were not enriched for KS- 
262 MVs. The lack of KS-MVs in these regions could be due to the lack of power of our study.

263 Alternatively, these variants may result in dominant-negative or gain-of-function effects,

264 specific to some cancers. We, therefore, specifically looked at the type of cancers reported

265 with Cancer-MVs in the FYR-C domain and between residues 3 043-3 248 (No domain \#8).

266 This showed that $87 \%$ and $82.1 \%$ of the variants detected in the FYR-C domain and No

267 domain \#8 regions came from solid cancers, but there was no enrichment for a specific type

268 of cancer (Table S3). Another possibility is that germline MVs in this region may result in a

269 condition different from KS, which has yet to be delineated.

270

$271460 / 570(80.7 \%)$ Cancer-MVs were outside the regions of the protein with statistically

272 significant clustering. Interestingly, 84/460 Cancer-MVs are part of set of overlapping

273 Cancer-MVs and Control-MVs in comparison with only 7/110 Cancer-MVs in the cancer-

274 enriched regions of KMT2D (Table S3-1). Overall, this analysis suggests that a substantial

275 number of these Cancer-MVs, which lie outside the cancer-enriched regions of KMT2D, may

276 not be driver variants but passengers ones.

277

278 For KS-MVs we detected significant clustering in the Coiled-coil\#5 and FYR-N domains,

279 and in between residue numbers 4 995-5 090 (No Domains \#16 in Figure S1), but we did not

280 identify significant clustering in these regions for Cancer-MVs. As MVs in these three

281 regions are likely to result in loss-of-function, the lack of Cancer-MVs in these regions is

282 likely to be due to lack of statistical power.

283

284 120/190 KS-MVs were outside the regions of the protein with statistically significant

285 clustering. Of note, 75/120 KS-MVs were also seen in Control-MVs in comparison with only

$28612 / 70 \mathrm{KS}-\mathrm{MVs}$ in the KS-enriched regions of KMT2D (Table S3-1). Furthermore, 107/120 
287 MVs were either inherited from an apparently unaffected parent or the information on

288 inheritance was unavailable. Taken together, $75 \mathrm{KS}-\mathrm{MVs}$ can be classed as benign or variants

289 of uncertain significance when classified according to the American College of Medical

290 Genetics guidelines (69). Finally, the misdiagnosis of KS in some patients might also explain

291 that their phenotypes do not match with their genetic findings, which may be benign.

292 Unfortunately, many KS-MVs were got from sources without a comprehensive individual

293 delineation of the syndrome, and most of those patients were just described as suffering from

294 KS (e.g. ClinVar, Hannibal et al. (12); Van Laarhoven et al. (30)). Therefore, we could not

295 filter patients with a true KS phenotype from those without it.

29722 MVs were seen in both KS and cancers (Table S3-1). Of note, 21 of these were present in

298 KS-enriched and/or Cancer-enriched regions. The unique MV that was not part of any of

299 these enriched regions, the p.Arg5340Leu substitution, may abolish the interaction between

300 KMT2D and WDR5 resulting in the complete loss of the H3K4 dimethylation activity of the

301 complex $(33,34)$. Thus, all the overlapping KS-MVs and Cancer-MVs are highly likely to be 302 pathogenic.

303

304 We did not find clustering of pathogenic MVs in a number of recognised domains and motifs

305 in KMT2D such as the SPPPEPEA region, the HMG Box, most coiled-coils (except coiled-

306 coil\#5), the LXXLL motifs and the Post-SET domains. The SPPPEPEA region is a poorly

307 characterised sequence of repeats composed by the amino acids Serine (S), Proline (P),

308 Glutamic acid (E) and Alanine (A) (70). The HMG (High mobility group) Box is a sequence

309 of $\sim 75$ amino acids that binds DNA (71). The LXXLL (L, Leucine; X, any amino acid)

310 motifs are necessary to activate nuclear receptors, and therefore, to activate transcription (72).

311 The Post-SET domain also contributes to the methyltransferase activity of KMT2D (68). Our 
312 results suggest that these regions of KMT2D are more tolerant to variations or that there may

313 be as yet unrecognised phenotypes associated with variants in these regions.

315 We found that the Cancer-MVs and KS-MVs tend to affect more conserved residues, KS-

316 MVs increase the energy that the protein needs for folding/interacting, and that Cancer-MVs

317 have a greater probability of disrupting protein interactions. We did not identify significant

318 difference in the ELASPIC $\Delta \Delta G$ scores or StructMAn scores of Cancer-MVs or KS-MVs

319 against Control-MVs, respectively (Figure 4B and C), which could be due to limited

320 available information on dynamics and interaction sites of KMT2D. This is reflected by our

321 observations that the ELASPIC $\Delta \Delta G$ scores and StructMAn interaction scores could be

322 generated for only 222/2 279 MVs and 92/2 279 MVs, respectively. This also limited the

323 analysis of scores according to the locations (e.g. the enriched regions) as most of these

324 values were given for the catalytic, PHD-1 and PHD-2 Zinc fingers domains only (Table S3).

325

326 Although this approach needs confirmation by large-scale functional analyses, which are

327 being described just recently (73), and a better characterisation of the protein structure of

328 KMT2D, a recent study about functional consequences of some MVs in this gene confirms

329 our methodology. Cocciadiferro et al. (34) demonstrated that MVs detected in patients with

$330 \mathrm{KS}$ and located on PHD-type zinc fingers \#3 and \#4 (p.Glu1391Lys, pMet1417Val,

331 p.Ile1428Thr, p.Ser1476Cys), RING-type zinc finger \#4 (p.Thr5098Pro), FYR-N

332 (p.Gly5189Arg, p. Trp5217Met) and SET (p.Arg5471Met, p.Glu5425Lys, p. Arg5471Met,

333 p.Tyr5510Asp) domains, and in between residue numbers 4 995-5 090 (No Domain \#16;

334 p.Phe5034Val, p.His5059Pro) decreased catalytic activity and/or disrupt the interaction of

335 KMT2D with ASH2L/RbBP5. These are exactly the same regions and domains that our study

336 found to be enriched in KS-MVs when compared to Control-MVs. Two exceptions are PHD- 
type zinc finger \#5 and Coiled-coil\#5 domains. While the p.Gln1522Arg MV in the former also disrupted enzymatic activity and interaction with $\mathrm{ASH} 2 \mathrm{~L} / \mathrm{RbBP} 5$, this domain was not detected to be enriched in KS-MVs in our analysis. This may be explained by the lack of enough MVs detected in patients with KS in this domain. Inversely, no MVs in Coiled-coil\#5 were studied by Cocciadiferro et al. (34), which cannot discard this domain as relevant for the

342 function of KMT2D.

344 Similarly, few Cancer-MVs have been characterised functionally and those findings are also concordant with our results. Zhang et al. (74) demonstrated that MVs detected in patients with lymphomas and located on RING-type zinc finger \#4 (p.Cys5092Ser, p.Cys5092Tyr), FYR-C (p.Asp5257Val) and SET (p.Arg5432Trp, p.Asn5437Ser, p.Gly5467Asp) domains decreased catalytic activity of KMT2D. These three domains were found to be enriched in Cancer-MVs when compared to Control-MVs. Other relevant MVs that decreased KMT2D activity in lymphomas were p.Arg5027Leu and p.Leu5056, which are located between residue numbers 4 995-5 090 (No Domain \#16). This region was not detected to be enriched in Cancer-MVs in our analysis, which may be explained by the type of cancer studied. Inversely, no MVs in PHD-type zinc fingers \#3 and \#4, and between residue numbers 3 0433248 (No domain \#8) were studied by Zhang et al. (74), which cannot discard these domains as relevant for the function of KMT2D.

In conclusion, this compilation can aid analysis of $K M T 2 D \mathrm{MVs}$ in diagnostic laboratories.

358 We show that rarity of KMT2D variants has limited value in determination of their

359 pathogenicity. We have identified a set of recurrent KMT2D MVs in cancer and KS. We

360 show that some presumed KMT2D MVs are in fact likely to result in loss of function by

361 introduction of frameshift. This work leads to reclassification of a set of presumed pathogenic 
362 MVs as benign variants or as VUS. We identify regions of the KMT2D protein that

363 demonstrate significant clustering of MVs in cancer and KS within and outside the known

364 domains and regions of the protein. We establish that the mechanism of most pathogenic

$365 K M T 2 D$ Cancer-MVs is loss of function, although other possibilities cannot be ruled out for

366 some atypical Cancer-MVs. We raise the possibility of as yet unrecognised 'non-KS'

367 phenotypes associated with some germline pathogenic MVs. Finally, this work provides

368 insights into the disease mechanism of cancers driven by KMT2D mutations and of KS1

369 (Kabuki syndrome type 1). Future work will be needed to understand the impact of the MVs

370 that could not be examined by the described in-silico programmes. Similar analyses in other

371 genes, mutations in which also cause developmental syndromes and cancer, should also be

372 carried out in the future $(1,2)$.

\section{Acknowledgments}

374 Víctor Faundes acknowledges to CONICYT, Chile's National Commission for Scientific and 375 Technological Research, for its scholarship support (grant number 72160007). All the authors

376 acknowledge to the Kabuki Research Fund at Manchester University NHS Foundation Trust.

\section{Disclosure statement}

378 The authors declare no conflict of interest. 


\section{References}

1. Faundes V, Newman WG, Bernardini L, Canham N, Clayton-Smith J, Dallapiccola B, et al. Histone Lysine Methylases and Demethylases in the Landscape of Human Developmental Disorders. American journal of human genetics. 2018;102(1):175-87.

2. Copeland RA. Protein methyltransferase inhibitors as precision cancer therapeutics: a decade of discovery. Philos Trans R Soc Lond B Biol Sci. 2018;373(1748).

3. Issaeva I, Zonis Y, Rozovskaia T, Orlovsky K, Croce CM, Nakamura T, et al. Knockdown of ALR (MLL2) reveals ALR target genes and leads to alterations in cell adhesion and growth. Molecular and cellular biology. 2007;27(5):1889-903.

4. Ng SB, Bigham AW, Buckingham KJ, Hannibal MC, McMillin MJ, Gildersleeve HI, et al. Exome sequencing identifies MLL2 mutations as a cause of Kabuki syndrome. Nature genetics. 2010;42(9):790-3.

5. Banka S, Veeramachaneni R, Reardon W, Howard E, Bunstone S, Ragge N, et al. How genetically heterogeneous is Kabuki syndrome?: MLL2 testing in 116 patients, review and analyses of mutation and phenotypic spectrum. European journal of human genetics : EJHG. 2012;20(4):381-8.

6. Banka S, Howard E, Bunstone S, Chandler KE, Kerr B, Lachlan K, et al. MLL2 mosaic mutations and intragenic deletion-duplications in patients with Kabuki syndrome. Clinical genetics. 2013;83(5):467-71.

7. Bogershausen N, Wollnik B. Unmasking Kabuki syndrome. Clinical genetics. 2013;83(3):201-11.

8. Ford DJ, Dingwall AK. The cancer COMPASS: navigating the functions of MLL complexes in cancer. Cancer genetics. 2015;208(5):178-91.

9. Rao RC, Dou Y. Hijacked in cancer: the KMT2 (MLL) family of methyltransferases. Nat Rev Cancer. 2015;15(6):334-46. 
10. Guo C, Chen LH, Huang Y, Chang CC, Wang P, Pirozzi CJ, et al. KMT2D maintains neoplastic cell proliferation and global histone H3 lysine 4 monomethylation. Oncotarget. 2013;4(11):2144-53.

11. Kim JH, Sharma A, Dhar SS, Lee SH, Gu B, Chan CH, et al. UTX and MLL4 coordinately regulate transcriptional programs for cell proliferation and invasiveness in breast cancer cells. Cancer research. 2014;74(6):1705-17.

12. Hannibal MC, Buckingham KJ, Ng SB, Ming JE, Beck AE, McMillin MJ, et al. Spectrum of MLL2 (ALR) mutations in 110 cases of Kabuki syndrome. American journal of medical genetics Part A. 2011;155A(7):1511-6.

13. Li Y, Bogershausen N, Alanay Y, Simsek Kiper PO, Plume N, Keupp K, et al. A mutation screen in patients with Kabuki syndrome. Human genetics. 2011;130(6):715-24.

14. Micale L, Augello B, Fusco C, Selicorni A, Loviglio MN, Silengo MC, et al. Mutation spectrum of MLL2 in a cohort of Kabuki syndrome patients. Orphanet journal of rare diseases. 2011;6:38.

15. Paulussen AD, Stegmann AP, Blok MJ, Tserpelis D, Posma-Velter C, Detisch Y, et al. MLL2 mutation spectrum in 45 patients with Kabuki syndrome. Human mutation. 2011;32(2):E2018-25.

16. Kokitsu-Nakata NM, Petrin AL, Heard JP, Vendramini-Pittoli S, Henkle LE, dos Santos DV, et al. Analysis of MLL2 gene in the first Brazilian family with Kabuki syndrome. American journal of medical genetics Part A. 2012;158A(8):2003-8.

17. Tanaka R, Takenouchi T, Uchida K, Sato T, Fukushima H, Yoshihashi H, et al. Congenital corneal staphyloma as a complication of Kabuki syndrome. American journal of medical genetics Part A. 2012;158A(8):2000-2.

18. Zarate YA, Zhan H, Jones JR. Infrequent Manifestations of Kabuki Syndrome in a Patient with Novel MLL2 Mutation. Molecular syndromology. 2012;3(4):180-4. 
19. Ito N, Ihara K, Tsutsumi Y, Miyake N, Matsumoto N, Hara T. Hypothalamic pituitary complications in Kabuki syndrome. Pituitary. 2013;16(2):133-8.

20. Miyake N, Koshimizu E, Okamoto N, Mizuno S, Ogata T, Nagai T, et al. MLL2 and KDM6A mutations in patients with Kabuki syndrome. American journal of medical genetics Part A. 2013;161A(9):2234-43.

21. Makrythanasis P, van Bon BW, Steehouwer M, Rodriguez-Santiago B, Simpson M, Dias P, et al. MLL2 mutation detection in 86 patients with Kabuki syndrome: a genotypephenotype study. Clinical genetics. 2013;84(6):539-45.

22. Cheon CK, Sohn YB, Ko JM, Lee YJ, Song JS, Moon JW, et al. Identification of KMT2D and KDM6A mutations by exome sequencing in Korean patients with Kabuki syndrome. J Hum Genet. 2014;59(6):321-5.

23. Giordano P, Lassandro G, Sangerardi M, Faienza MF, Valente F, Martire B.

Autoimmune haematological disorders in two Italian children with Kabuki syndrome. Italian journal of pediatrics. 2014;40:10.

24. Micale L, Augello B, Maffeo C, Selicorni A, Zucchetti F, Fusco C, et al. Molecular analysis, pathogenic mechanisms, and readthrough therapy on a large cohort of Kabuki syndrome patients. Human mutation. 2014;35(7):841-50.

25. Dentici ML, Di Pede A, Lepri FR, Gnazzo M, Lombardi MH, Auriti C, et al. Kabuki syndrome: clinical and molecular diagnosis in the first year of life. Archives of disease in childhood. 2015;100(2):158-64.

26. Lin JL, Lee WI, Huang JL, Chen PK, Chan KC, Lo LJ, et al. Immunologic assessment and KMT2D mutation detection in Kabuki syndrome. Clinical genetics. 2015;88(3):255-60.

27. Lindsley AW, Saal HM, Burrow TA, Hopkin RJ, Shchelochkov O, Khandelwal P, et al. Defects of B-cell terminal differentiation in patients with type-1 Kabuki syndrome. The Journal of allergy and clinical immunology. 2015. 
28. Liu S, Hong X, Shen C, Shi Q, Wang J, Xiong F, et al. Kabuki syndrome: a Chinese case series and systematic review of the spectrum of mutations. BMC medical genetics. 2015;16:26.

29. Morgan AT, Mei C, Da Costa A, Fifer J, Lederer D, Benoit V, et al. Speech and language in a genotyped cohort of individuals with Kabuki syndrome. American journal of medical genetics Part A. 2015;167(7):1483-92.

30. Van Laarhoven PM, Neitzel LR, Quintana AM, Geiger EA, Zackai EH, Clouthier DE, et al. Kabuki syndrome genes KMT2D and KDM6A: functional analyses demonstrate critical roles in craniofacial, heart and brain development. Human molecular genetics.

2015;24(15):4443-53.

31. Yuen RK, Thiruvahindrapuram B, Merico D, Walker S, Tammimies K, Hoang N, et al. Whole-genome sequencing of quartet families with autism spectrum disorder. Nature medicine. 2015;21(2):185-91.

32. Forbes SA, Beare D, Gunasekaran P, Leung K, Bindal N, Boutselakis H, et al. COSMIC: exploring the world's knowledge of somatic mutations in human cancer. Nucleic acids research. 2015;43(Database issue):D805-11.

33. Shinsky SA, Hu M, Vought VE, Ng SB, Bamshad MJ, Shendure J, et al. A nonactive-site SET domain surface crucial for the interaction of MLL1 and the RbBP5/Ash2L heterodimer within MLL family core complexes. Journal of molecular biology. 2014;426(12):2283-99.

34. Cocciadiferro D, Augello B, De Nittis P, Zhang J, Mandriani B, Malerba N, et al. Dissecting KMT2D missense mutations in Kabuki syndrome patients. Human Molecular Genetics. 2018:ddy241-ddy. 
35. Aref-Eshghi E, Schenkel LC, Lin H, Skinner C, Ainsworth P, Pare G, et al. The defining DNA methylation signature of Kabuki syndrome enables functional assessment of genetic variants of unknown clinical significance. Epigenetics-Us. 2017;12(11):923-33.

36. Sobreira N, Brucato M, Zhang L, Ladd-Acosta C, Ongaco C, Romm J, et al. Patients with a Kabuki syndrome phenotype demonstrate DNA methylation abnormalities. European journal of human genetics : EJHG. 2017;25(12):1335-44.

37. Velankar S, van Ginkel G, Alhroub Y, Battle GM, Berrisford JM, Conroy MJ, et al. PDBe: improved accessibility of macromolecular structure data from PDB and EMDB. Nucleic acids research. 2016;44(D1):D385-95.

38. Davies H, Bignell GR, Cox C, Stephens P, Edkins S, Clegg S, et al. Mutations of the BRAF gene in human cancer. Nature. 2002;417(6892):949-54.

39. Sarkozy A, Carta C, Moretti S, Zampino G, Digilio MC, Pantaleoni F, et al. Germline BRAF mutations in Noonan, LEOPARD, and cardiofaciocutaneous syndromes: molecular diversity and associated phenotypic spectrum. Human mutation. 2009;30(4):695-702.

40. Ramos P, Karnezis AN, Craig DW, Sekulic A, Russell ML, Hendricks WP, et al. Small cell carcinoma of the ovary, hypercalcemic type, displays frequent inactivating germline and somatic mutations in SMARCA4. Nature genetics. 2014;46(5):427-9.

41. Tsurusaki Y, Okamoto N, Ohashi H, Kosho T, Imai Y, Hibi-Ko Y, et al. Mutations affecting components of the SWI/SNF complex cause Coffin-Siris syndrome. Nature genetics. 2012;44(4):376-8.

42. Reijnders MRF, Ansor NM, Kousi M, Yue WW, Tan PL, Clarkson K, et al. RAC1 Missense Mutations in Developmental Disorders with Diverse Phenotypes. American journal of human genetics. 2017;101(3):466-77.

43. Menke LA, study DDD, Gardeitchik T, Hammond P, Heimdal KR, Houge G, et al. Further delineation of an entity caused by CREBBP and EP300 mutations but not resembling 
Rubinstein-Taybi syndrome. American journal of medical genetics Part A. 2018;176(4):86276.

44. Cuvertino S, Stuart HM, Chandler KE, Roberts NA, Armstrong R, Bernardini L, et al. ACTB Loss-of-Function Mutations Result in a Pleiotropic Developmental Disorder. American journal of human genetics. 2017;101(6):1021-33.

45. Riviere JB, van Bon BW, Hoischen A, Kholmanskikh SS, O'Roak BJ, Gilissen C, et al. De novo mutations in the actin genes ACTB and ACTG1 cause Baraitser-Winter syndrome. Nature genetics. 2012;44(4):440-4, S1-2.

46. Seiler M, Peng S, Agrawal AA, Palacino J, Teng T, Zhu P, et al. Somatic Mutational Landscape of Splicing Factor Genes and Their Functional Consequences across 33 Cancer Types. Cell reports. 2018;23(1):282-96 e4.

47. Lek M, Karczewski KJ, Minikel EV, Samocha KE, Banks E, Fennell T, et al. Analysis of protein-coding genetic variation in 60,706 humans. Nature. 2016;536(7616):28591.

48. 1000 Genomes Project Consortium, Auton A, Brooks LD, Durbin RM, Garrison EP, Kang HM, et al. A global reference for human genetic variation. Nature. 2015;526(7571):6874.

49. Sherry ST, Ward M, Sirotkin K. dbSNP-database for single nucleotide polymorphisms and other classes of minor genetic variation. Genome research. 1999;9(8):677-9.

50. Fu W, O'Connor TD, Jun G, Kang HM, Abecasis G, Leal SM, et al. Analysis of 6,515 exomes reveals the recent origin of most human protein-coding variants. Nature. 2013;493(7431):216-20.

51. Stenson PD, Mort M, Ball EV, Shaw K, Phillips A, Cooper DN. The Human Gene Mutation Database: building a comprehensive mutation repository for clinical and molecular 
genetics, diagnostic testing and personalized genomic medicine. Human genetics. 2014;133(1):1-9.

52. Landrum MJ, Lee JM, Benson M, Brown G, Chao C, Chitipiralla S, et al. ClinVar: public archive of interpretations of clinically relevant variants. Nucleic acids research. 2016;44(D1):D862-8.

53. McLaren W, Gil L, Hunt SE, Riat HS, Ritchie GR, Thormann A, et al. The Ensembl Variant Effect Predictor. Genome biology. 2016;17(1):122.

54. Raponi M, Kralovicova J, Copson E, Divina P, Eccles D, Johnson P, et al. Prediction of single-nucleotide substitutions that result in exon skipping: identification of a splicing silencer in BRCA1 exon 6. Human mutation. 2011;32(4):436-44.

55. Cerami E, Gao J, Dogrusoz U, Gross BE, Sumer SO, Aksoy BA, et al. The cBio cancer genomics portal: an open platform for exploring multidimensional cancer genomics data. Cancer discovery. 2012;2(5):401-4.

56. Gao J, Aksoy BA, Dogrusoz U, Dresdner G, Gross B, Sumer SO, et al. Integrative analysis of complex cancer genomics and clinical profiles using the cBioPortal. Science signaling. 2013;6(269):pl1.

57. Henikoff S, Henikoff JG. Amino acid substitution matrices from protein blocks. Proceedings of the National Academy of Sciences of the United States of America. 1992;89(22):10915-9.

58. Berliner N, Teyra J, Colak R, Garcia Lopez S, Kim PM. Combining structural modeling with ensemble machine learning to accurately predict protein fold stability and binding affinity effects upon mutation. PloS one. 2014;9(9):e107353.

59. Gress A, Ramensky V, Buch J, Keller A, Kalinina OV. StructMAn: annotation of single-nucleotide polymorphisms in the structural context. Nucleic acids research. 2016;44(W1):W463-8. 
60. Li Y, Han J, Zhang Y, Cao F, Liu Z, Li S, et al. Structural basis for activity regulation of MLL family methyltransferases. Nature. 2016;530(7591):447-52.

61. Garcia-Alai MM, Allen MD, Joerger AC, Bycroft M. The structure of the FYR domain of transforming growth factor beta regulator 1. Protein Sci. 2010;19(7):1432-8.

62. Truebestein L, Leonard TA. Coiled-coils: The long and short of it. Bioessays. 2016;38(9):903-16.

63. Carlston CM, O'Donnell-Luria AH, Underhill HR, Cummings BB, Weisburd B, Minikel EV, et al. Pathogenic ASXL1 somatic variants in reference databases complicate germline variant interpretation for Bohring-Opitz Syndrome. Human mutation. 2017;38(5):517-23.

64. Dhar SS, Lee SH, Kan PY, Voigt P, Ma L, Shi XB, et al. Trans-tail regulation of MLL4-catalyzed H3K4 methylation by H4R3 symmetric dimethylation is mediated by a tandem PHD of MLL4. Gene Dev. 2012;26(24):2749-62.

65. Ali M, Hom RA, Blakeslee W, Ikenouye L, Kutateladze TG. Diverse functions of PHD fingers of the MLL/KMT2 subfamily. Biochimica et biophysica acta. 2014;1843(2):366-71.

66. Sanchez R, Zhou MM. The PHD finger: a versatile epigenome reader. Trends in biochemical sciences. 2011;36(7):364-72.

67. Borden KL, Freemont PS. The RING finger domain: a recent example of a sequencestructure family. Current opinion in structural biology. 1996;6(3):395-401.

68. Zhang Y, Mittal A, Reid J, Reich S, Gamblin SJ, Wilson JR. Evolving Catalytic Properties of the MLL Family SET Domain. Structure. 2015;23(10):1921-33.

69. Richards S, Aziz N, Bale S, Bick D, Das S, Gastier-Foster J, et al. Standards and guidelines for the interpretation of sequence variants: a joint consensus recommendation of the American College of Medical Genetics and Genomics and the Association for Molecular 
Pathology. Genetics in medicine : official journal of the American College of Medical Genetics. 2015;17(5):405-24.

70. UniProt Consortium. O14686 (KMT2D_HUMAN) 1998 [updated 17 February 2016. Available from: http://www.uniprot.org/uniprot/O14686.

71. Malarkey CS, Churchill ME. The high mobility group box: the ultimate utility player of a cell. Trends in biochemical sciences. 2012;37(12):553-62.

72. Savkur RS, Burris TP. The coactivator LXXLL nuclear receptor recognition motif. J Pept Res. 2004;63(3):207-12.

73. Findlay GM, Daza RM, Martin B, Zhang MD, Leith AP, Gasperini M, et al. Accurate classification of BRCA1 variants with saturation genome editing. Nature. 2018.

74. Zhang J, Dominguez-Sola D, Hussein S, Lee JE, Holmes AB, Bansal M, et al. Disruption of KMT2D perturbs germinal center B cell development and promotes lymphomagenesis. Nature medicine. 2015;21(10):1190-8. 


\section{Figure Legends}

\subsection{Figure 1: Study design}

Summary of steps followed for compilation and analysis of missense variants (MV) in KMT2D.

\subsection{Figure 2: Presumed KMT2D MVs that are likely to disrupt splicing are enriched in Kabuki syndrome and cancer}

Variants affecting the first or last three bases of exons (first/last in red, second/second last in orange and third/third last in green) are depicted. Variants seen in Kabuki syndrome are denoted with *, variants seen in cancer are denoted with \#, and are placed above the transcript (ENST00000301067.11), whereas control variants are placed below the transcript. The proportion of presumed MVs predicted to affect splicing is significantly higher for KS-MVs

and Cancer-MVs in comparison with Control-MVs $\left(\chi^{2}=21.88, \mathrm{df}=2, \mathrm{p}=0.000018\right)$. Within the variants predicted to disrupt splicing, a further enrichment of canonical splice-donor and splice-acceptor sites can be found in cancer and KS (variants in red). Interestingly, the six Control-MVs affecting the canonical splice-donor and splice-acceptor sites either do not increase the probability of exon-skipping or are predicted to result in in-frame exon skipping.

\subsection{Figure 3: Specific regions of the KMT2D protein are enriched for missense variants in Kabuki syndrome and cancer}

Distributions of KMT2D missense variants (MV) seen in (A) control population, (B) cancers, and $(\mathrm{C})$ Kabuki syndrome (KS) is shown. The X-axis shows the length of the KMT2D protein and the location of its domains and regions. The domains and regions are color-coded 
and the legend is provided at the bottom of the figure. The enriched regions/domains in cancers or in Kabuki syndrome are highlighted in red brackets in the respective panels. The $\mathrm{Y}$-axis in (A) shows minor allele frequencies of controls and in (B and C) the number of times a specific Cancer-MV or KS-MV was seen in our cohort. (D) Proportion of KMT2D missense variants grouped according to domains and regions.

\subsection{Figure 4: Cancer and Kabuki syndrome MVs affect more conserved residues, increase KMT2D delta-delta free energy and may disrupt its interaction with other proteins.}

Global comparisons of (A) BLOSUM62, (B) ELASPIC $\Delta \Delta \mathrm{G}$ and(C) StructMAn scores of missense variants (MV) seen in control population, cancers and Kabuki syndrome. When compared to Control-MVs, Cancer-MVs and KS-MVs have both significantly lower BLOSUM scores, KS-MVs have significantly higher ELASPIC $\Delta \Delta \mathrm{G}$ scores, and CancerMVs have significantly higher StructMAn scores. 


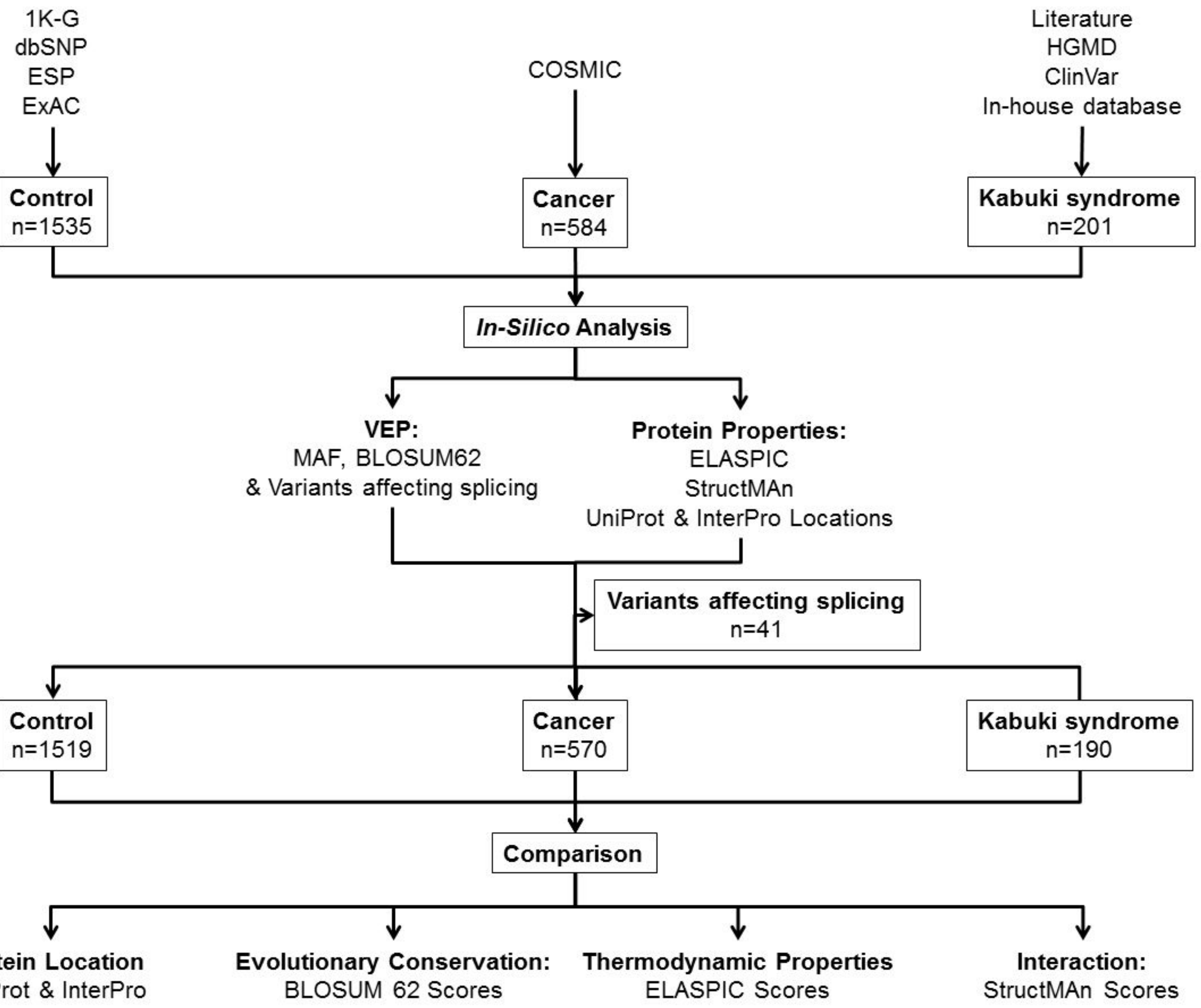




\section{Exon 4:}

c. $510 \mathrm{G}>C^{*}$

c. $4131 \mathrm{G}>\mathrm{C}^{*}$

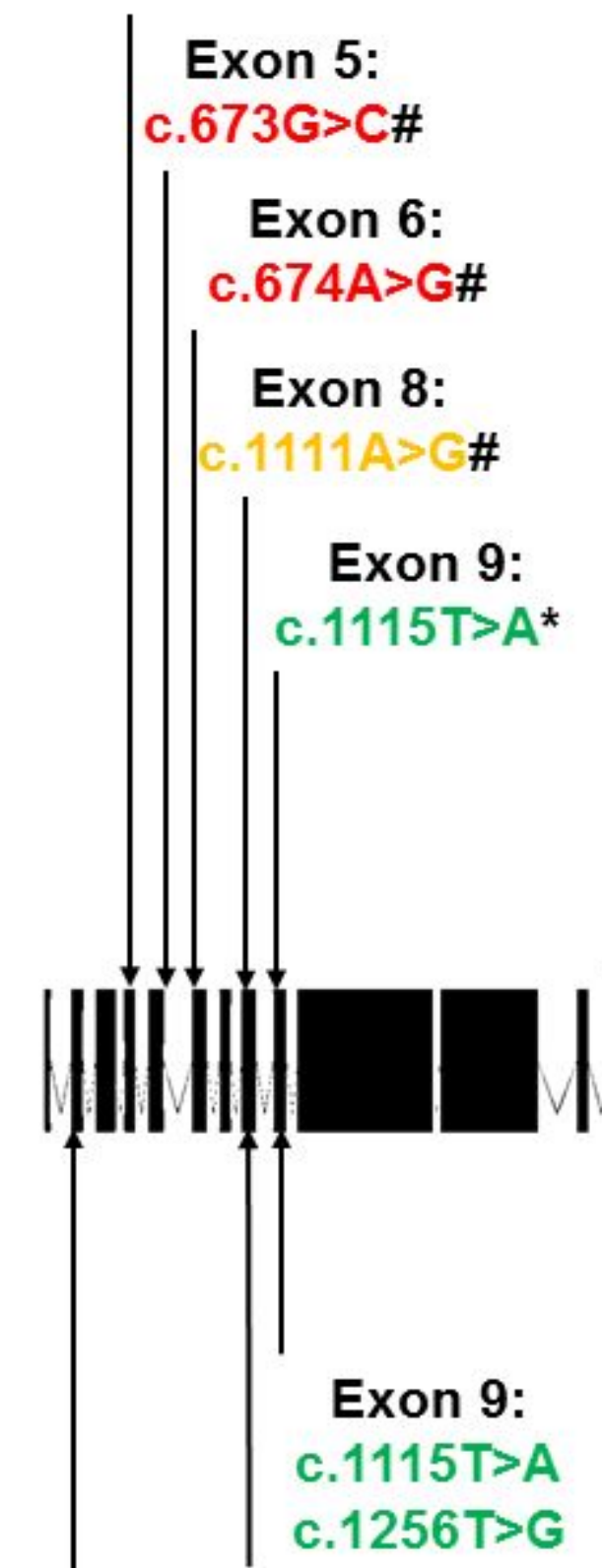

\section{Exon 8:}

c. $956 \mathrm{C}>\mathrm{T}$

\section{Exon 2:}

c. $50 \mathrm{C}>\mathrm{T}$

c. $52 \mathrm{G}>\mathrm{T}$
Exon 14:

c. $4235 \mathrm{~A}>\mathrm{G}$ \#

Exon 16:

c. $4421 \mathrm{G}>\mathrm{T \#}$

c. $4421 \mathrm{G}>\mathrm{A}^{*}$

Exon 19:

c. $4963 \mathrm{G}>\mathrm{A \#}$

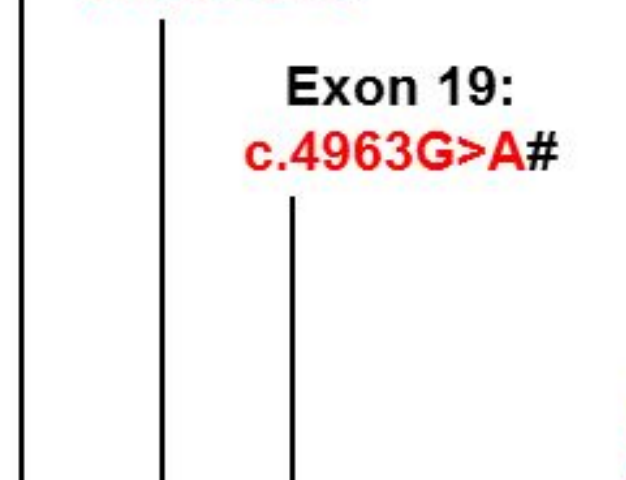

Exon 51:

c. $16338 \mathrm{G}>\mathrm{C} \#$
Exon 45:

c. 14254T>A\#

c. $14381 A>G$ *

Exon 33:

c. $8366 \mathrm{G}>\mathrm{A}^{*}$ c. $8366 \mathrm{G}>\mathrm{A}$ \#

\section{Exon 36:}

Exon 41: c.13672G>C\#

c.10439A>T\# c. $10440 \mathrm{G}>\mathrm{T} \#$
Exon 52:

c. $16341 \mathrm{~T}>\mathrm{G} \#$

c. $16411 \mathrm{~A}>\mathrm{T}^{*}$

c. $16412 \mathrm{G}>\mathrm{T}^{*}$ c. $16412 \mathrm{G}>\mathrm{C}^{*}$

Exon 53: c. $16413 \mathrm{G}>\mathrm{T}^{*}$

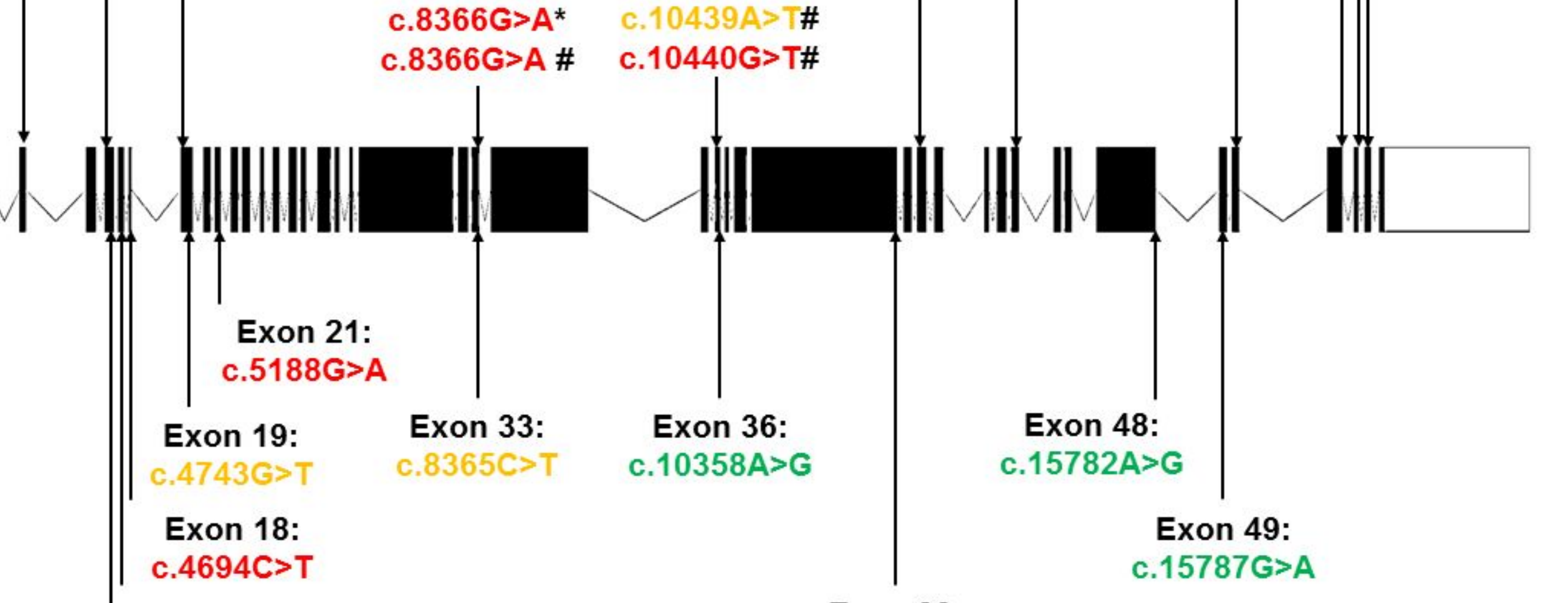

Exon 17:

c. $4693 \mathrm{G}>\mathrm{T}$

Exon 16:

c. $4419 \mathrm{G}>\mathrm{T}$
Exon 39:

c. $13528 \mathrm{G}>\mathrm{C}$

Exon 13:

c. $4131 \mathrm{G}>\mathrm{C}$ 


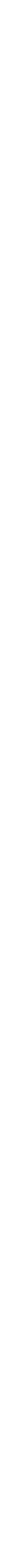



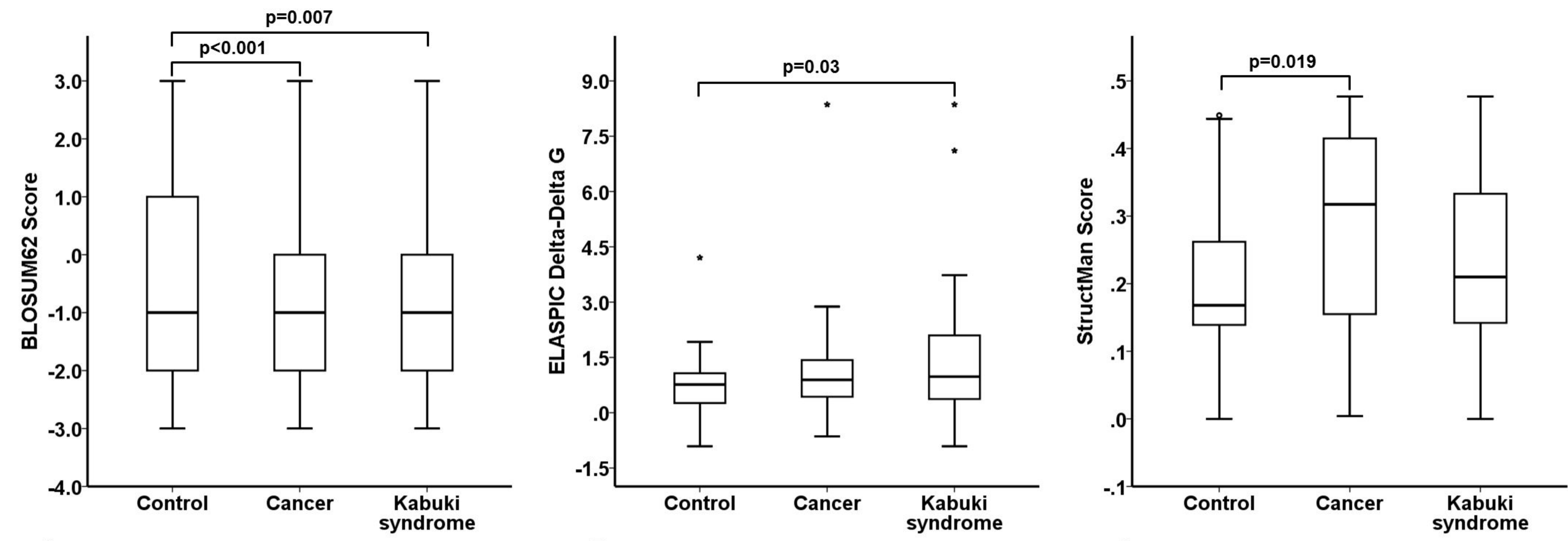

A

B

C 
Table 1. Comparison of proportions of missense variants seen in control population, cancer and Kabuki syndrome according to their grouped locations.

\begin{tabular}{|c|c|c|c|}
\hline Protein Domain $^{a}$ & $\begin{array}{c}\text { Control Population } \\
\begin{array}{c}\text { n (\%) } \\
(n=1519)\end{array}\end{array}$ & $\begin{array}{l}\text { Cancer } \\
\text { n (\%) } \\
(n=570)\end{array}$ & $\begin{array}{c}\text { Kabuki syndrome } \\
\text { n (\%) } \\
(n=190)\end{array}$ \\
\hline RING-type Zinc Finger & $20(1.3)$ & $13(2.3)$ & $6(3.2)$ \\
\hline PHD-type Zinc Finger & $40(2.6)$ & $40(7.0)^{b}$ & $27(14.2)^{C}$ \\
\hline SPPPEPEA region & $90(5.9)$ & $27(4.7)$ & $4(2.1)$ \\
\hline HMG Box & $10(0.7)$ & $8(1.4)$ & $1(0.5)$ \\
\hline Coiled coil & $27(1.8)$ & $12(2.1)$ & $4(2.1)$ \\
\hline LXLL motif & $15(1)$ & $3(0.5)$ & $0(0)$ \\
\hline FYR-N Terminal & $27(1.8)$ & $12(2.1)$ & $13(6.8)^{b}$ \\
\hline FYR-C Terminal & $10(0.7)$ & $23(4)^{b}$ & $3(1.6)$ \\
\hline SET & $6(0.4)$ & $22(3.9)^{b}$ & $12(6.3)^{b}$ \\
\hline Post-SET & $0(0)$ & $1(0.2)$ & $0(0)$ \\
\hline No Domain & 1274 (83.9) & $409(71.8)^{d}$ & $120(63.2)^{d}$ \\
\hline \multicolumn{4}{|c|}{$\begin{array}{l}\text { a In order of location; domains with significantly different pr } \\
\text { phenotypes (p-adjusted<0.05) are depicted in italic. } \\
\text { b Proportion significantly higher than controls. } \\
{ }^{\text {c }} \text { Proportion significantly higher than the other two groups. } \\
\text { d Proportion significantly lower than controls. }\end{array}$} \\
\hline
\end{tabular}


Table 2. Comparison of proportions of missense variants seen in control population, cancer and Kabuki syndrome according to every significantly different location.

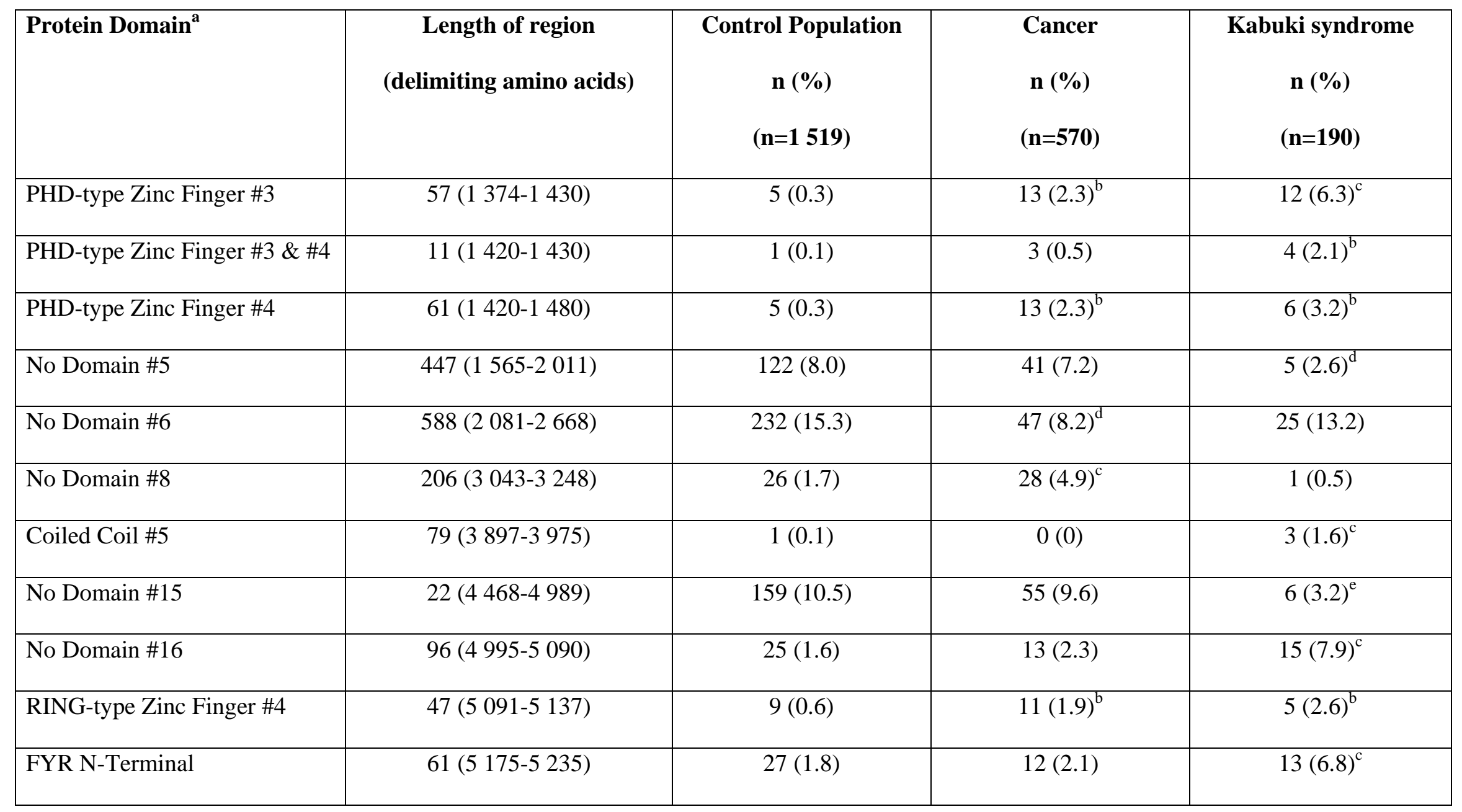




\begin{tabular}{|c|c|c|c|c|}
\hline FYR C-Terminal & $92(5$ 236-5 327) & $10(0.7)$ & $23(4.0)^{b}$ & $3(1.6)$ \\
\hline \multicolumn{5}{|c|}{${ }^{\mathrm{a}}$ In order of location; only domains with significantly different proportions amongst the phenotypes (p-adjusted $<0.05$ ) are depicted. For } \\
\hline \multicolumn{5}{|c|}{ visualisation of these regions, see Figure S2. } \\
\hline \multicolumn{5}{|c|}{ c Proportion significantly higher than the other two groups. } \\
\hline \multicolumn{5}{|c|}{ d Proportion significantly lower than controls. } \\
\hline e Proportion signi & other two groups. & & & \\
\hline
\end{tabular}

